\title{
Keep calm and... essere un Nefrologo!
}

\section{Gaetano Lucisano}

Post-CCT Fellow in Renal Medicine, West London Renal and Transplant Centre, Imperial College Healthcare NHS Trust, London - UK

\begin{abstract}
Keep calm and... be a Nephrologist!
A working experience abroad is an educational pearl in the specialist training process, a unique occasion to call into question and enrich one's own knowledge. I think that the young "Nephrologists-to-be" should be encouraged and supported as much as possible to spend abroad some of their training time. Beyond the current issue of the medical "brain drain", to set a foot outside the country is always instructive, and I herein describe my personal experience.
\end{abstract}

Keywords: Training, Abroad, Experience

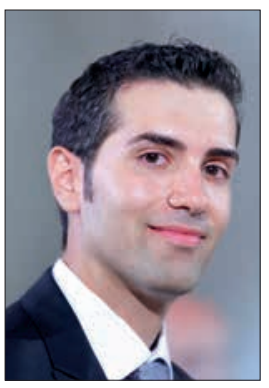

Gaetano Lucisano

\section{Introduzione}

Un periodo trascorso all'estero è un elemento di grande utilità nella formazione specialistica di un medico; ne arricchisce il bagaglio culturale e conferisce una particolare "apertura mentale" che scaturisce dal confronto con altre culture e modi di fare. È un'ottima occasione per mettersi in discussione: confrontare le proprie "skill" con quelle del luogo, affinandole, scambiando conoscenze e idee e arricchendosi sempre come risultato finale. Ciò permette di fare il secondo passo, ovviamente per chi è interessato: lavorare all'estero. Lo sappiamo bene, al giorno d'oggi sempre più medici italiani, specialisti e non, decidono di intraprendere la loro carriera lavorativa oltre frontiera: Svizzera, Francia, Germania, Regno Unito... Naturalmente, lascio a voi la riflessione sul possibile motivo per il quale oggi un giovane medico debba salutare Terra, amici e famiglia, mentre io preferisco raccontarvi in poche righe la mia esperienza.

Accepted: October 13, 2015

Published online: November 19, 2015

Indirizzo per la corrispondenza:

Dr. Gaetano Lucisano

40 Holborn House

Du Cane Road

W12 0TS

London, United Kingdom

gaetano_lucisano@hotmail.com

\section{La mia prima esperienza all'estero: la Germania}

Ho studiato medicina all'Università di Catanzaro, dove mi sono specializzato in Nefrologia nel 2014, e da oltre un anno lavoro come Trust Doctor presso la Nefrologia dell'Imperial College Healthcare NHS Trust di Londra. Quando ero quasi alla fine del mio secondo anno di Specializzazione, alla luce del dimostrato interesse per la ricerca, il Direttore della Scuola, Prof. Giorgio Fuiano, mi spinse a "lanciarmi" in un'esperienza Erasmus. Scegliemmo come sede del mio stage la Germania, presso l'Unità di Nefrologia del Policlinico di Homburg, Università del Saarland, ancora oggi diretta dal Prof. Danilo Fliser. Quest'ultimo mi accolse calorosamente e dimostrò fin da subito ampia disponibilità, assegnandomi all'Unità di Ultrasonografia del suo Dipartimento e al gruppo di ricerca del Prof. Gunnar Heine, con il quale ho stretto un'amicizia e una collaborazione professionale sincere che continuano tutt'oggi. Durante tale stage, un semestre in tutto, ebbi la grande occasione di imparare a eseguire l'ecografia e l'ecoduplex su rene e arterie renali nel nativo e nel trapiantato, oltre che in altri distretti vascolari periferici e centrali. Presi, inoltre, parte agli studi che venivano condotti nel gruppo di ricerca del Prof. Heine, tra i quali uno studio prospettico sulla CKD-MBD nei pazienti emodializzati presso il Policlinico di Homburg.

\section{Ritorno a casa e la fine della specializzazione: di cosa ho ancora bisogno?}

Al mio ritorno ebbi la fortuna di poter continuare a praticare l'ecoduplex. Oltre a ciò, iniziai ben presto a interessarmi al mondo dell'istopatologia renale, che veniva praticata di continuo all'interno della Scuola di Catanzaro. Così, ancora una volta, il Professor Fuiano mi diede, nel 2012, una grande opportunità: partecipare all'International Summer School of Renal Pathology, 
diretta dal Prof. Loreto Gesualdo, che ogni anno ospita insegnanti di fama mondiale esperti in istopatologia renale.

Ricerca clinica, ultrasonografia, istopatologia... ma ancora mancava un tassello: il trapianto! Come fare? II tempo passava in fretta, avevo "giocato" la mia carta Erasmus e organizzare nuovi spostamenti in convenzione richiedeva del tempo. L'ultimo anno di Specializzazione era, peraltro, iniziato, periodo in cui ci si capisce che bisogna iniziare a metter su un piano per la "fine". E come sarebbe stata la mia "fine"? Quanto avrei dovuto attendere per la pubblicazione di un concorso/avviso pubblico... e sarebbe andato a buon fine, data l'ingente mole di Nefrologi "a caccia" di un posto di lavoro sul territorio italiano? Avevo 30 anni e desideravo tanto continuare a imparare e a fare esperienze "speciali", oltre al fatto che sentivo di voler approfondire le mie conoscenze sul trapianto. Dovevo, quindi, ritentare la strada dell'estero e senza alcun supporto, poiché, stavolta, non puntavo a uno stage, bensì a un incarico di lavoro. Optai per il Regno Unito e alcuni mesi prima di concludere la Specializzazione avviai la procedura per iscrivermi al General Medical Council, requisito indispensabile per poter svolgere l'attività medica nel Regno Unito. Per coloro che hanno conseguito la laurea nell'Unione Europea, la procedura non è molto farraginosa, sebbene la traduzione dei vari documenti richieda del tempo. Dopo circa 5 mesi ottenni, così, l'abilitazione all'esercizio della Professione nel Regno Unito e subito dopo sottomisi la mia application per una posizione di Clinical Fellow in Renal Medicine presso l'Imperial College NHS Trust di Londra. Due settimane dopo la mia seduta di Specializzazione ricevetti l'email che mi comunicava che il mio $\mathrm{CV}$ era stato selezionato e, dopo quattro giorni, ero a Londra per svolgere il colloquio e muovere i primi passi in una delle più grandi unità renali in Europa.

\section{West London renal and transplant centre e I'NHS}

II West London Renal and Transplant Centre (WLRTC) ha sede all'Hammersmith Hospital, uno degli ospedali che fanno parte dell'Imperial College Healthcare NHS Trust. Si tratta delI' "hub nefrologico" di Londra a ovest del Tamigi: 60 posti letto di degenza ordinaria, 15 di degenza sub-intensiva, 9 unità dialisi satellite con circa 2500 pazienti in terapia sostitutiva e ambulatori specialistici in 7 ospedali di Londra Ovest. Ogni anno circa 200 pazienti vengono trapiantati all'Hammersmith Hospital (rene, pancreas e rene-pancreas) e, attualmente, quasi 2000 trapiantati di rene vengono seguiti negli ambulatori dell'Hammersmith e del St. Mary's Hospital. II WLRTC ha, all'attivo, il più grande programma di trapianto di rene ABOincompatibile nel Regno Unito. Lavorare al WLRTC mi ha permesso di imparare tantissimo non solo in tema di trapianto, ma anche sulla gestione delle glomerulonefriti, in particolare delle vasculiti ANCA-associate e della nefrite lupica. Infatti, si sa che quest'ultima ha un'elevata incidenza nella popolazione di etnia afro-caraibica, particolarmente rappresentata in Inghilterra, ma, soprattutto, a Londra.

Imparare a muoversi in un nuovo ambiente di lavoro non è facile all'inizio... e se ciò rientra in un nuovo Paese e in un nuovo sistema sanitario e ospedaliero, il tutto è ancora più difficile! ... E non sei più "visitor", ma un dipendente pubblico a tutti gli effetti! Ma la medicina è la stessa in tutto il mondo e, come ho già detto, mettersi in discussione (per chi ha l'umiltà di farlo...) è sempre costruttivo... e, allora, si impara in fretta a essere efficienti al massimo.

La classica domanda che mi è spesso stata posta è: quale sistema sanitario preferisci, quello inglese o quello italiano? $\mathrm{Si}$ tratta di due sistemi validi e sostanzialmente diversi, pertanto non mi sento di metterli a confronto. Un sistema sanitario fornisce servizi sulla base delle caratteristiche e delle problematiche tipiche di una popolazione e quella inglese, ma, in particolare, quella londinese, differisce significativamente da quella italiana: basti solo pensare alla multietnicità e al bacino di utenza (per esempio, Londra ha 6 unità renali per quasi $9 \mathrm{mi}-$ lioni di abitanti). Cambiano, pertanto, le gerarchie e i compiti nel personale medico e infermieristico, la presa in carico del paziente sul territorio e la gestione delle informazioni cliniche.

\section{Conclusioni}

Svolgere l'attività medica in realtà grandi come quella in cui mi trovo oggi richiede impegno e sacrificio, ma, d'altro canto, ampie casistiche permettono di fare molta esperienza, che, alla mia età e per un medico specialista, è di importanza e utilità estreme. Oggi, dopo un anno in cui ho lavorato come Clinical Fellow, ho ottenuto all'Imperial College NHS Trust la posizione di Post-CCT Fellow, che mi permette, oltre all'attività clinica, di fare ricerca nel gruppo di studio di cui attualmente faccio parte, quello sul trapianto renale, diretto dal Professor David Taube.

Classica domanda finale: "Ritorneresti in Italia"? II mio Paese e i miei affetti mi mancano ogni giorno, sarebbe anormale se così non fosse. Non credete a chi verrà a dirvi che espatriare è tutto "rose e fiori": ogni scelta ha il suo prezzo, insieme alle sue ricompense. II posto dove oggi $\mathrm{mi}$ trovo $\mathrm{mi}$ sta insegnando ogni giorno tantissimo, il che è una fortuna. Tuttavia, non escludo un giorno di ritornare "in patria": dipenderà dalle circostanze e, soprattutto, dall'eventualità che il mio trascorso oltremanica possa suscitare dell'interesse.

Riguardo ai giovani aspiranti Nefrologi, un semplice messaggio: credete nelle vostre capacità e siate sempre bramosi di nuove conoscenze! Partite, se potete, anche per un breve periodo: vi aiuterà a capire come potervi migliorare e vi aprirà la mente. Spero che la Società Italiana di Nefrologia possa sostenere con sempre maggiore impegno la buona volontà degli aspiranti Nefrologi che vogliono affacciarsi sull'Europa. E, per coloro che partiranno... non dimenticate mai il profumo e i colori della vostra Terra!

\section{Disclosures}

Financial support: No financial support was received for this submission.

Conflict of interest: The author has no conflict of interest. 